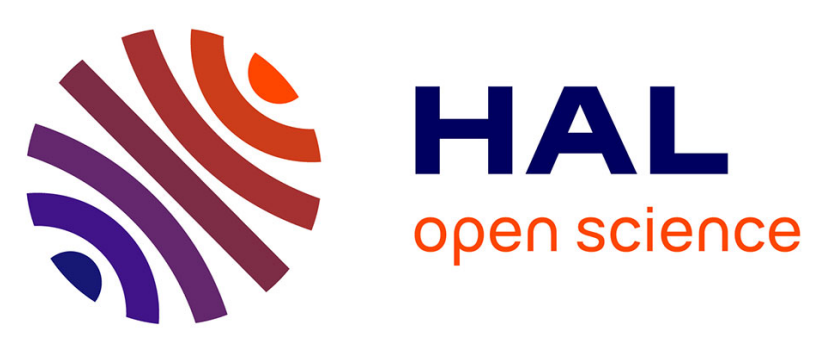

\title{
A novel approach to relax extremely localized molecular orbitals: the extremely localized molecular orbital-valence bond method
}

\author{
Alessandro Genoni, M. Sironi
}

\section{- To cite this version:}

Alessandro Genoni, M. Sironi. A novel approach to relax extremely localized molecular orbitals: the extremely localized molecular orbital-valence bond method. Theoretical Chemistry Accounts: Theory, Computation, and Modeling, 2004, 112 (4), pp.254-262. 10.1007/s00214-004-0585-0 . hal-02196446

\section{HAL Id: hal-02196446 \\ https://hal.univ-lorraine.fr/hal-02196446}

Submitted on 27 May 2020

HAL is a multi-disciplinary open access archive for the deposit and dissemination of scientific research documents, whether they are published or not. The documents may come from teaching and research institutions in France or abroad, or from public or private research centers.
L'archive ouverte pluridisciplinaire HAL, est destinée au dépôt et à la diffusion de documents scientifiques de niveau recherche, publiés ou non, émanant des établissements d'enseignement et de recherche français ou étrangers, des laboratoires publics ou privés. 


\title{
A novel approach to relax extremely localized molecular orbital: the ELMO-VB method
}

\author{
Alessandro GenONI ${ }^{(\mathrm{A})}$ MAURIZIO SirONI ${ }^{(\mathrm{A}, \mathrm{B})^{*}}$ \\ (A) Dipartimento di Chimica Fisica ed Elettrochimica-Universita' di Milano \\ Via Golgi 19, 20133 Milano - Italy \\ (B) Centre for Bio-molecular Interdisciplinary Studies and Industrial Applications \\ CISI - Via Fratelli Cervi n. 93-Palazzo LITA 20090 Segrate (MI)- Italy \\ E-mail: maurizio.sironi@unimi.it \\ Tel.: +390250314251
}

\section{Abstract}

Orbitals rigorously defined on molecular fragments, like the extremely localized molecular orbitals, represent a possible way to build up the electronic structure of large molecules, using molecular fragments assembled on small molecules. Of course a rigorous localization is strictly connected to a reduction in the number of variational parameters, which reflects in an increasing value of the associated energy. In order to get both an extremely localized description of the molecule and an increase of the energetic accuracy, we have developed a method which allows a relaxation of the electronic structure based on localized orbitals. The relaxation is realized by means of a Valence Bond technique, which in turn uses the localized nature of the orbitals to reduce the number of excitations. Applications to the transferability of extremely localized molecular orbitals are presented.

Keywords: extremely localized molecular orbitals, transferability, VB approaches

\section{Introduction}

The theoretical development of methods allowing to study large molecules is still a challenging target for the computational chemist. The problems scientists have 
to deal with in the field of drug design or in the modelling related to the discovery of new materials ask for the application of traditional methods of computational chemistry to molecules of increasing complexity.

Molecular orbital (MO) methods have always had a prominent role in the framework of theoretical chemistry, and due to their orthogonality have permitted a huge development of many well known packages to determine the electronic structure of molecules with an increasing accuracy. For small molecules theoretical results have reached an accuracy which is comparable, and sometimes superior, to experimental values.

The application of theoretical chemistry to large molecules ask for methods which scale favourably with the increasing dimension of the system, and a lot of work has been done by different groups in order to build up the so called $\mathrm{N}$-linear methods $[1-4]$.

A possible approach to set up algorithms for the study of large molecules derives from two ideas that are well recognized by every chemist: the concepts of locality and transferability. When looking at a new molecule, our first step to rationalize its structure and properties is to look up the different functional groups which constitute it: we are looking for local entities. Once we have determined them, we start to do previsions on the molecule, as we already know those of its different functional groups: we are applying the transferability property.

Hence it is quite natural that theoreticians have tried to insert these ideas in the framework of different models. Traditional MO methods do not seem adequate to this purpose, owing to the delocalization of the canonical MOs on the whole system. Also a slight modification of the structure of the molecule, for example the addition of a methyl group, requires a complete redetermination of all the MOs of the molecule. In order to overcome these problems, different well known localization schemes have been proposed [5 - 7]. Due to the double occupancy of the MOs, it is in fact possible to transform them in different sets characterized by a different spatial extension, but with the same energy value.

In this way it is possible to define local MOs (LMOs) which allow to recover the local concepts; however, due to the orthogonal nature of the LMOs, they are characterized by the presence of tails which extend beyond the local region preventing a rigorous application of the transferability. 
Extremely Localized Molecular Orbitals (ELMOs) or Strictly Localized Molecular Orbitals (SLBOs) [8 - 9] or Non-orthogonal Localized Molecular Orbitals (NLMO) [10] are molecular orbitals which are developed on only the atomic centres of pre-selected molecular fragments and, in this way, they have rigorously no tails on the other atomic centres. This is easily obtained defining for each ELMO a proper Linear Combination of Atomic Orbitals (LCAO) on only the atoms of its molecular fragment.

The ELMOs are of course closely connected to the group function method introduced by McWeeny [11], which can be considered as one of the first theoretical attempts to describe a wavefunction through functions associated to subsets of electrons. The ELMOs are not orthogonal and, even if their definition and the theory to determine them has been set up many years ago [12], recently there has been a renewed interest in their determination and use [13 - 16]. The algorithms to obtain ELMOs are not trivial, as it is well known that serious convergence difficulties arise. Couty et al. [16] have explicitly treated the nonorthogonality using the Löwdin formula for the computation of the nonorthogonal matrix elements. To reduce the computational cost associated to the evaluation of the Hessian matrix, they computed its diagonal blocks using an approximate formula, while for the other blocks they used an updated scheme based on the Davidon-Fletcher-Powell or Broyden-Fletcher-Goldfarb-Shanno methods. Very recently, Surjan et al. [15] have proposed a method based on the use of appropriate projection operators to determine ELMOs.

In our laboratory we have developed a method [13] based on the generalization of the equations proposed by Gianinetti et al. [17] for the Self-Consistent Field for Molecular Interactions (SCF-MI). Very recently, we have proposed another approach [14] based on the equations by Stoll [12] and we are now extending it to the Density Functional Theory [18]. After a long work in the convergence routines, we think to have realized a robust algorithm, which up to now has always converged also when using some well known delicate localization schemes, such as an extremely localized description of the $\pi$ system of benzene. We have tested the transferability of the ELMOs [19] showing that electronic properties of some ortho-substituted biphenyl molecules determined at the Hartree Fock level are quite well reproduced optimizing only the ELMOs defined on the substituent group, while keeping all the other ELMOs identical to the 
corresponding ELMOs determined for the unsubstituted biphenyl molecule. We have also compared the different transfer ability of ELMOs with respect to tails deleted LMOs in reproducing electrostatic properties of acetone [14] determined at the Hartree Fock level.

From these works it was evident that the ELMOs provide results of superior quality with respect to the LMOs, as one could easily guess due to the non negligible perturbation introduced by the tails deletion. It was also evidenced that a very close agreement between the results obtained with transferred ELMOs and the Hartree-Fock ones can be obtained with just a simple relaxation, such as that provided by a single SCF iteration using the transferred ELMOs as starting orbitals.

The ELMOs could also be a valuable tool to define frontier region in the mixed Quantum Mechanics/ Molecular Mechanics methods based on the use of localized MOs to describe the covalent bonds which connect quantum and classical regions, according to the Local Self Consistent Field proposed by Rivail et al. [20 - 21].

It is quite evident that the price to pay in order to have rigorously defined ELMOs is a loss in the number of variational parameters that we use to describe them. This has a quite evident effect on the energy values associated to the ELMO wavefunction, which are significantly higher with respect to the RHF values, but also significantly lower with respect to those obtained using LMOs after tails deletion. Therefore, it appears quite important to develop methods which, starting from the ELMOs approach, try to improve the wavefunction using cheap methods. One could guess that the extremely localized nature of the orbitals could help in this effort, similarly to the good efficiency provided by the local MPn methods [22] which use the local nature of the orbitals to efficiently determine correlation energies.

In this paper, we propose a novel approach to relax ELMOs based on a very compact VB expansion of the wavefunction, which allows the occupied ELMOs to delocalize on the near fragments, so recovering a good percentage of energy with respect to the Hartree-Fock wavefunction. Test calculations have been performed together with an exemplification in the use of transferred ELMOs with the present approach. It will be also shown that the localized nature of the orbitals 
permits to greatly reduce the VB expansion, so posing the prerequisites to use the present approach for studying large molecules.

The proposed method can be considered as a natural development of the Perturbative Configuration Interaction using localised orbitals (PCILO) [23-24] and of the studies carried out to reduce the CI expansion using LMOs [25].

\section{Theory}

Let us consider a closed shell molecule. ELMOs are defined by partitioning the $N$ MOs (where $2 N$ is the total number of the electrons) into $n_{f}$ molecular fragments,

where each of them is defined by its own basis set, $\left(\chi_{\mu}^{i}\right)_{\mu=1}^{n_{i}} m_{i}$ being the number of the atomic orbitals belonging to the $i$-th fragment.

The $\alpha$-th ELMO of the $i$-th fragment can thus be written as:

$$
\varphi_{\alpha}^{i}=\sum_{\mu}^{m_{i}} c_{\mu \alpha}^{i} \chi_{\mu}^{i}
$$

The coefficients $c_{\mu \alpha}^{i}$ are determined through a minimization of the expectation value of the energy associated to the ELMO wavefunction:

$$
\psi_{E L M O}=A(\Phi) \quad \text { with } \Phi=\prod_{i}^{n_{f}} \prod_{\alpha}^{N_{i}^{o}} \varphi_{\alpha}^{i} \overline{\varphi_{\alpha}^{i}}
$$

where $A$ is the antisymmetrizer operator and $N_{i}^{o}$ is the number of doubly occupied ELMOs belonging to the $i$-th fragment.

It should be noted that fragments can share atomic orbitals, then a natural nonorthogonality between ELMOs of different fragments arises. Different algorithms to determine ELMOs have been proposed both in the past and recently [12-16]. It should be observed that the non-orthogonality of the orbitals causes non trivial converge difficulties.

The limitations introduced in the expansion definition of the ELMOs causes an obvious increment of the energy associated to the ELMOs wavefunction with respect to the Hartree-Fock one. It is expected that this penalty should be larger when ELMOs are determined on smaller molecules and then transferred on a larger one without optimization. Hence it appears of relevant interest the 
development of strategies to relax the ELMOs wavefunction to obtain an energy lowering with respect to the value associated to a straightforward use of the ELMOs.

To overcome the limitations connected to the expansion definition of the ELMOs, they should be combined, once the convergence is reached, with the virtual orbitals of the other fragments in order to gain an energy lowering. Linear combinations of occupied orbitals of a fragment with their own virtual orbitals cannot give a lowering in energy due to the Brillouin theorem, and a combination between doubly occupied orbitals of different fragments cannot be useful because of the Pauli principle.

Hence, in order to obtain a relaxation of the ELMOs wavefunction, we must permit the occupied ELMOs of a fragment to delocalize using the virtual ELMOs of the other fragments. In other words, we allow an interaction between the ELMOs wavefunction and a number of structures derived from it exciting the electrons from the occupied ELMOs to the virtual ones. Due to the non orthogonal nature of the ELMOs, their configuration interaction can be thought as a VB expansion:

$$
\Psi_{E L M O-V B}=c_{0} \Psi_{E L M O}+\sum_{i r}^{n_{f}} \sum_{\alpha}^{N_{i}^{o}} \sum_{\mu}^{N_{r}^{v}} c_{\alpha \mu}^{i r} \Psi_{\alpha \mu}^{i r}+\ldots .
$$

where $N_{r}^{v}$ is the number of virtual ELMOs of the $r$-th fragment, $c_{\alpha \mu}^{i r}$ is the weight of the structure $\Psi_{\alpha \mu}^{i r}$ which is a single excitation from the $\alpha$-th ELMO of the $i$-th fragment to the $\mu$-th virtual orbital of the $r$-th fragment, and it is expressed by:

$$
\Psi_{\alpha \mu}^{i r}=A\left(\Phi\left(\begin{array}{l}
i \\
\alpha
\end{array}\right) \varphi_{\alpha}^{i} \varphi_{\mu}^{r} \Theta_{0,0}^{2}\right)
$$

where $\Phi\left(\begin{array}{l}i \\ \alpha\end{array}\right)$ is the product of all the occupied ELMOs with the exception of the pair $\varphi_{\alpha}^{i} \overline{\varphi_{\alpha}^{i}}$, i.e. $\Phi=\Phi\left(\begin{array}{l}i \\ \alpha\end{array}\right) \varphi_{\alpha}^{i} \overline{\varphi_{\alpha}^{i}}$ (in a similar way the symbol $\Phi\left(\begin{array}{c}i j \\ \alpha \beta\end{array}\right)$ is defined by $\left.\Phi=\Phi\left(\begin{array}{c}i \mathrm{j} \\ \alpha \beta\end{array}\right) \varphi_{\alpha}^{i} \overline{\varphi_{\alpha}^{i}} \varphi_{\beta}^{j} \overline{\varphi_{\beta}^{j}}\right)$ and $\Theta_{0,0}^{2}$ is the singlet spin eigenfunction for the electrons belonging to the orbitals $\varphi_{\alpha}^{i} \varphi_{\mu}^{r}$.

Of course the expansion of eq. (1) could be extended to higher orders of excitations, but we have to consider that here we are just looking for a relaxation of the occupied ELMOs and that higher excitations would bring us to a very 
demanding algorithm, vanishing the advantages to use an approach based on localized orbitals.

For this reason we will limit expansion (1) to single excitations. It should be recalled that in this approach, in opposition to a traditional MO-CI, single excitations give a contribution to the energy as each ELMO has been obtained using only its own basis set.

Due to the non orthogonal nature of the orbitals, the computation of the hamiltonian matrix elements $\left\langle\Psi_{\alpha \mu}^{i r}|H| \Psi_{\beta v}^{j s}\right\rangle$ should require the use of the Löwdin formula [26] for a system of $2 \mathrm{~N}$ electrons, so the calculation would soon become prohibitive.

In order to reduce the computational effort, the orbitals are subjected to the following transformations:

a) the occupied ELMOs are subjected to a Löwdin orthogonalization giving rise to a new set of occupied ELMOs $\left(\overline{\overline{\varphi_{\alpha}^{i}}}\right)_{i=1 ; \alpha=1}^{n_{f} ; N_{i}^{o}}$

b) each virtual orbital $\varphi_{\mu}^{r}$ is subjected to a Gram-Schmidt orthogonalization with respect to the Löwdin-orthogonalized occupied ELMOs, obtaining the orbitals $\left(\overline{\overline{\varphi_{\beta}^{r}}}\right)_{r=1 ; \beta=1}^{n_{f} ; N_{i}^{v}}$

It should be noted that when eq. (1) involves all the single excitations to the selected virtual orbitals, the results obtained with the orthogonalized orbitals are identical to those obtained with the starting non-orthogonal ELMOs.

So a VB calculation is performed using the ELMO wavefunction $\overline{\bar{\Psi}}_{\text {ELMO }}$ built up using the occupied $\overline{\overline{\varphi_{\alpha}^{i}}}$ orbitals and the single excitations $\overline{\overline{\Psi_{\alpha \mu}^{i r}}}$ in the set of the Gram-Schmidt orthogonalized virtual orbitals:

$$
\Psi_{E L M O-V B}=\overline{\overline{c_{0}}} \overline{\overline{\Psi_{E L M O}}}+\sum_{i r}^{n_{f}} \sum_{\alpha}^{N_{i}^{o}} \sum_{\mu}^{N_{r}^{v}} \overline{\overline{c_{\alpha \mu}^{i r}}} \overline{\overline{\Psi_{\alpha \mu}^{i r}}}
$$

It should be observed that step b) does not introduce an orthogonalization between the virtual orbitals, which remain non-orthogonal. In this way a partial localization nature of the virtual orbitals is preserved. 
In order to perform the VB calculation, it is necessary to compute the matrix elements of the hamiltonian operator between the structures appearing in the VB expansion (2).

Due to the orthogonality between the virtual orbitals and the occupied ones, the calculation of these matrix elements can be greatly simplified [27] through a straightforward manipulation of the Löwdin formula. For example, the matrix elements between a couple of single excitations, i.e. $\left\langle\overline{\overline{\Psi_{\alpha \mu}^{i r}}}|H| \overline{\overline{\Psi_{\beta v}^{j s}}}\right\rangle$, can be expressed in the following way:

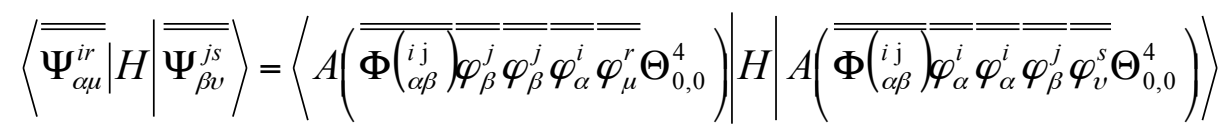

where the symbol $\overline{\overline{\Phi\left(\begin{array}{l}i j \\ \alpha \beta\end{array}\right)}}$ has been already defined and it is here applied to the $\overline{\overline{\varphi_{\alpha}^{i}}}$ orbitals, and $\Theta_{0,0}^{4}=\alpha \beta \Theta_{0,0}^{2}$ is the spin function.

The structures contained in the bra and ket parts of the previous equation are characterized by a common set of $N^{\prime}=N-2$ doubly occupied orbitals described by

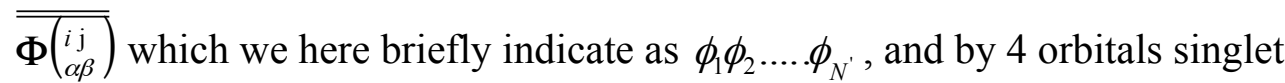
paired and orthogonal to the $\overline{\overline{\Phi\left(\begin{array}{c}i \mathrm{j} \\ \alpha \beta\end{array}\right)}}$ alignment.

We will indicate with $\left(\zeta_{r}^{a}\right)_{r=1}^{4}$ and $\left(\zeta_{s}^{b}\right)_{s=1}^{4}$ the orbitals $\overline{\overline{\varphi_{\beta}^{j}}} \overline{\overline{\varphi_{\beta}^{j}}} \overline{\overline{\varphi_{\alpha}^{i}}} \overline{\overline{\varphi_{\mu}^{r}}}$ and $\overline{\overline{\varphi_{\alpha}^{i}}} \overline{\overline{\varphi_{\alpha}^{i}}} \overline{\overline{\varphi_{\beta}^{j}}}=\overline{\overline{\varphi_{v}^{s}}}$ and with $\psi_{a}$ and $\psi_{b}$ the single excitations $\overline{\overline{\Psi_{\alpha \mu}^{i r}}}$ and $\overline{\overline{\Psi_{\beta v}^{j s}}}$, respectively. The hamiltonian matrix element of eq. (3) is given by:

$$
\begin{aligned}
& \left\langle\Psi_{a}|H| \Psi_{b}\right\rangle=2 D_{a b} \sum_{k=1}^{N^{\prime}}\left\langle\phi_{k}|h| \phi_{k}\right\rangle+D_{a b} \sum_{k, h=1}^{N^{\prime}} 2\left\langle\phi_{k} \phi_{k} \| \phi_{h} \phi_{h}\right\rangle-\left\langle\phi_{k} \phi_{h} \| \phi_{h} \phi_{k}\right\rangle+ \\
& \sum_{r, s=1}^{4}\left\langle\zeta_{r}^{a}\left|h^{e f f}\right| \zeta_{s}^{b}\right\rangle D_{a b}[r \mid s]+ \\
& \sum_{r<s}^{4} \sum_{t<u}^{4}\left\langle\zeta_{r}^{a} \zeta_{t}^{b} \| \zeta_{s}^{a} \zeta_{u}^{b}\right\rangle D_{a b}[r s \mid t u]-\sum_{r<s}^{4} \sum_{t<u}^{4}\left\langle\zeta_{r}^{a} \zeta_{u}^{b} \| \zeta_{s}^{a} \zeta_{t}^{b}\right\rangle D_{a b}[r s \mid u t]
\end{aligned}
$$

where $\langle a b \| c d\rangle=\iint d \mathbf{r}_{1} d \mathbf{r}_{\mathbf{2}} a\left(\mathbf{r}_{\mathbf{1}}\right) b\left(\mathbf{r}_{\mathbf{1}}\right) \frac{1}{\left|\mathbf{r}_{\mathbf{1}}-\mathbf{r}_{\mathbf{2}}\right|} c\left(\mathbf{r}_{\mathbf{2}}\right) d\left(\mathbf{r}_{\mathbf{2}}\right), D_{a b}, D_{a b}[r \mid s]$,

$D_{a b}[r s \mid t u]$ are the elements of the transition density matrices with respect to zero, 
one and two particles [11], respectively, and $h^{\text {eff }}$ operator gives the effect of the electrons described by $\overline{\overline{\Phi\left(\begin{array}{l}i \mathrm{j} \\ \alpha \beta\end{array}\right)}}$ on the other electrons.

Eq. (4) can be efficiently programmed, considering that the terms which explicitly take into account the non-orthogonality of the orbitals are characterized by summations with indexes ranging from 1 to 4 . The remaining terms contain expressions with indexes ranging from 1 to $N$, but as they do not vary significantly when changing the excitations, it is possible to compute them by appropriate differences with respect to the value obtained with $N^{\prime}=N$. For example the first term of equation (4), apart from the trivial multiplicative factor $D_{a b}$, can be computed through:

$$
\sum_{k=1}^{N}\left\langle\phi_{k}|h| \phi_{k}\right\rangle-\left\langle\varphi_{\alpha}^{i}|h| \varphi_{\alpha}^{i}\right\rangle-\left\langle\varphi_{\beta}^{j}|h| \varphi_{\beta}^{j}\right\rangle
$$

where the first term is independent from the pairs of excitations considered. Using the present approach it is possible to obtain an algorithm which allows to save more than one order of CPU time with respect to a direct application of the Löwdin formula.

Particular care has to be dedicated to the selection of the virtual orbitals that have to be introduced in the VB expansion. We have to recall that in the ELMOs approach we obtain $m_{i}$ orbitals for each fragment; as the fragments can share the same atomic basis functions, it results that $\sum_{i}^{n_{f}} m_{i}>M$, where $M$ is the total number of the basis functions. This fact does not cause any problem in the determination of the ELMOs, but requires a particular attention in the VB expansion, as linear dependence problems can easily arise if no criteria are introduced in the selection of the virtual orbitals.

As a matter of fact we noted that it is quite common to find virtual ELMOs of a fragment which are linear combinations of the occupied ELMOs of the other fragments. In this case these orbitals are automatically removed by our procedure during step b) where they are Gram-Schmidt orthogonalized with respect to the occupied orbitals.

Of course this procedure does not guarantee that the survival virtual orbitals are not linearly dependent to each other. In order to be sure to select a proper linear independent set of virtual orbitals, it is possible to submit the set of the virtual 
orbitals from step $\mathrm{b}),\left(\overline{\overline{\varphi_{\beta}^{r}}}\right)_{r=1 ; \beta=1}^{n_{f} ; N_{i}^{v}}$, to a Gram-Schmidt procedure obtaining a new set of orbitals which we denote $\left({ }^{G S} \varphi_{\beta}^{r}\right)_{r=1 ; \beta=1}^{n_{f} ; N_{i}^{v}}$. If the norm of the orbital ${ }^{G S} \varphi_{\beta}^{r}$ immediately after the Gram-Schmit orthogonalization step is greater than a given threshold, the original $\overline{\overline{\varphi_{\beta}^{r}}}$ orbital is added to the set of virtual orbitals that have to be included in the VB expansion.

The use of the set $\left(\overline{\overline{\varphi_{\beta}^{r}}}\right)_{r=1 ; \beta=1}^{n_{f} ; N_{i}^{v}}$ instead of $\left({ }^{G S} \varphi_{\beta}^{r}\right)_{r=1 ; \beta=1}^{n_{f} ; N_{i}^{v}}$ is due to the localized nature of the first one, that allows, as we will see in the examples reported in this work, to favourably truncate the VB expansion.

\section{Test calculations}

Some test calculations were performed to evaluate the proposed approach. The first one concerns the butane molecule, for which we have used the geometry optimized at the RHF level with the standard 6-31G basis set.

To perform the ELMOs calculation, we have defined the molecular fragments following the scheme depicted in Fig. 1.

\section{Fig. 1}

The fragments 1 to 4 correspond to the cores of the carbon atoms and are defined using only the atomic functions of their respective centres; the fragments $5,6,7,9,10,12,13,15,16,17$ describe the bonds $\mathrm{C}-\mathrm{H}$ and each of them is defined using the corresponding atomic functions of the carbon and hydrogen atoms describing that bond; finally the fragments 8,11,14 describe the $\mathrm{C}-\mathrm{C}$ bonds and each of them is defined according to the atomic functions of the two involved carbon atoms.

The RHF calculation has been performed using the PC-GAMESS package [28 29], which has also been used to obtain the mono- and bi-electronic integrals necessary to perform the ELMO-VB calculations; the ELMO calculations have been carried out using a modified version of the PC-GAMESS package [14], and 
for the ELMO-VB we used a program written in our laboratory to test the proposed method. It has been checked using a general purpose VB program [30]. The energy values are reported in Table 1.

\section{Table 1}

We can observe that due to the extremely localized nature of the orbitals, the energy corresponding to the ELMOs wavefunction differs by $27.9 \mathrm{Kcal} / \mathrm{mol}$ with respect to the RHF value. This difference is largely increased, up to 80.9 $\mathrm{Kcal} / \mathrm{mol}$, if we use a wavefunction built up using LMOs obtained trough the Pipek-Mezey procedure [7], subjected to a tails deletion of the components outside the molecular fragments defined in Figure 1.

We have then performed two ELMO-VB calculations, denoted with ELMO$\mathrm{VB}(1)$ and ELMO-VB(2), where one or two virtual ELMOs for each fragment have been selected, respectively. Due to the orthogonalization with respect to the occupied ELMOs, they acquire a partial delocalization with respect to the original virtual ELMOs, but anyway they conserve a quite stringent localized nature, as it can be seen in the examples reported in Fig. 2.

\section{Fig. 2}

The ELMO-VB(1) wavefunction based on the use of only one virtual orbital for each fragment is constituted by 289 excitations and it recovers $24 \%$ of the difference energy between the RHF and the ELMO wavefunctions.

The use of two virtual orbitals for each fragment allows the ELMO-VB(2) wavefunction to recover $84 \%$ of the difference energy, still remaining a compact wavefunction, as it is constituted by 578 excitations.

In order to see the contributions of the excitations to the ELMO-VB(1) and ELMO-VB(2) expansions, we have ordered the excitations according to their decreasing absolute weights and we have performed a series of ELMO-VB calculations introducing an increasing number of excitations according to their weights. In this way we can have an idea of the relative importance of the different excitations. 
In Fig. 3 the energy values of the different ELMO-VB wavefunctions are plotted against the number of excitations included into the VB expansion. The percentage results are reported in Fig. 4.

\section{Fig. 3}

\section{Fig. 4}

From Figs. 3 and 4 it is evident that not all the structures have the same importance in the ELMO-VB expansion. Roughly the same results of the final ELMO-VB wavefunction can be obtained with just half the number of total excitations. This result can be ascribed to the localized nature of all the orbitals involved in the VB expansion. The occupied orbitals, despite the Löwdin orthogonalization, remain well localized, and the virtual ones acquire a partial but very limited delocalization after the Gram-Schmidt orthogonalization. These considerations suggest that the VB expansion can be compacted taking into account the local nature of the orbitals [23-25].

In order to further investigate this point we have performed two additional ELMO-VB calculations, where we have selected only the following excitations:

a) from an occupied ELMO describing a bond (i.e. a C-C or a C-H fragment) to virtual ELMOs localized on the first neighbour fragments (e.g. from fragment 5 to 6 or from 5 to 1 ) or to the second neighbour fragments (e.g. from 5 to 9 or 5 to 2 ).

b) from an occupied ELMO describing a bond (C-C or C-H) to virtual ELMOs localized on the third neighbour core fragments (e.g. from 5 to 3 or 9 to 4$)$.

These are a sort of criteria based on what we could call 'a topological selection' aimed to insert in the VB expansion only the more interacting excitations. The energy values obtained with these calculations are reported in Table 1 . The ELMO-VB(Top_1) calculation using just a single virtual orbital for each fragment, is constituted by 166 excitations (i.e. $57 \%$ of the VB expansion with no 
topological selection), but it recovers $19.9 \%$ of the energy with respect to the Hartree Fock value, to compare with $24 \%$ if all the structures are included. The favourable comparison is more evident with the ELMO-VB(Top_2) wavefunction using two virtual ELMOs for each fragment and topological selection. In this case the wavefunction is constituted by 322 excitations (i.e. $56 \%$ with respect to the VB expansion with no topological selection), but it recovers $78.2 \%$ of the energy to compare with $84.2 \%$ without restrictions on the excitations included.

In order to study the applicability of the ELMO-VB method to the relaxation of the transferred ELMOs, we have considered the 3-pentanone molecule.

Using the optimized geometry at the RHF level with the 6-31G basis set, we have carried out an ELMOs calculation using the molecular fragments depicted in Figure 5.

\section{Figure 5 -}

The definitions of the fragments is similar to those adopted for the butane molecule. In this case we observe that the fragment 4, defined using the atomic orbitals of the oxygen atom, is constituted by three occupied molecular orbitals, describing the core and the lone pairs electrons; the fragment 14 is built up using the atomic functions of the oxygen atom and of the bonded carbon atom, and it is constituted by two occupied molecular orbitals which describe the $\mathrm{C}=\mathrm{O}$ double bond.

\section{Table 2}

The energy values are reported in Table 2. Once again we observe that the energy associated to the ELMOs wavefunction is higher than the RHF value by 54.3 $\mathrm{Kcal} / \mathrm{mol}$. Anyway we note that the use of LMOs subjected to the tails deletion in order to reproduce the same molecular fragments definition of the ELMOs increases this difference up to $124.7 \mathrm{Kcal} / \mathrm{mol}$.

In order to investigate the transferability properties of the ELMOs, we have performed ELMOs calculations on the ethane, formaldehyde and acetaldehyde molecules, using the same definition of the molecular fragments adopted so far (i.e. bond and core/lone pair fragments). In this way we have defined ELMOs for 
all the molecular fragments which are present in the 3-pentanone molecule. The complete absence of tails permits a direct transferring of the ELMOs from the three smaller molecules to the 3-pentanone one (see Fig. 6)

\section{Fig. 6}

Using the transferred ELMOs we have determined the energy, ELMO-Transf, of the 3-pentanone molecule, which is only $2.7 \mathrm{Kcal} / \mathrm{mol}$ higher with respect to that achieved through optimized ELMOs. So the resulting difference with respect to the Hartree Fock value is $57.0 \mathrm{Kcal} / \mathrm{mol}$.

We have then performed ELMO-VB calculations using one/two, ELMO$\mathrm{VB}(1) /(2)$, virtual orbitals for each fragment. The ELMO-VB(1)/(2) expansion is so constituted by 504 (1008) excitations and allows to recover $44.0 \%(80.3 \%)$ of the energy difference between the RHF and the ELMOs values. It is quite satisfying that the use of transferred ELMOs and the ELMO-VB approach permit to obtain a wavefunction quite close to the RHF calculation on the 3-pentanone molecule. It should be observed that there are 1776 variational coefficients defining the 3-pentanone RHF wavefunction, and only 290 coefficients which define the ELMOs wavefunction.

In order to see if it is possible to reduce the VB expansion while retaining good accuracy, we have performed two additional ELMO-VB calculations using the 'topological selection' individuated for the butane molecule, the virtual orbitals of the oxygen lone pairs being treated as the virtual orbitals describing the bonds. The energy values are reported in Table 2. The two VB expansions, denoted as ELMO-VB(Top_1)/(Top_2) are now reduced to 224/448 excitations and they recover $30.4 \% / 73.5 \%$ of the energy difference between the RHF and ELMOs calculations. Hence it can be concluded that the 'topological selection' allows to halve the lengths of the VB expansion while only slightly affecting the accuracy of the results. 


\section{Conclusions}

The ELMOs provide a description of a molecule using variationally determined orbitals. Due to the large reduction of the variational coefficients, the ELMOs wavefunction is characterised by a non negligible increase of the energy. Anyway this is the price to pay in order to have localized MOs which are really transferable without perturbation. Of course a different approach could be to perform a posteriori localization of the canonical MOs, and to delete their tails before transferring them. However this procedure is characterised by higher increase in the energy, and we have recently evidenced [14] that also the electronic properties suffer from such approach.

It is therefore of relevant importance to develop methods which are based on the ELMOs, and allow, at the same time, to increase the accuracy of the electronic description of the system.

In this paper we have proposed a method based on a VB expansion of the ELMOs wavefunction. The localized nature of the orbitals has permitted to define a 'topological selection' of the excitations to include in the VB espansion, allowing to reduce the computational requests without substantial reduction in the accuracy of the results. The calculations have also evidenced that just two virtual orbitals for each fragment allow to obtain results close to the Hartree-Fock wavefunction. The same results are obtained using transferred ELMOs, so satisfying the basilar request for using this approach to describe large molecules.

In the present method we have strongly limited the VB expansion having truncated it to the single excitations. Even if this could be considered a severe approximation, we must recall that the aim of the present work is not to introduce correlation using localized orbitals, but to relax ELMOs in order to get results closer to the Hartree Fock ones with respect to those obtained by the ELMOs wavefunction. In addition it should be observed that the last objective of all the methods that try to assemble the electronic structure using molecular fragments should be the application to large molecules, so the number of higher excitations for these systems would become prohibitively expensive. 
References

1. Goedecker S, (1999) Rew. Mod. Phys. 71: 1085

2. Lee TS, York DM, Yang W, (1996) J. Chem. Phys. 105: 2744

3. Van der Vaart A, Gogonea V, Dixon SL, Merz KM Jr, (2000) J. Comput. Chem. 21: 1494

4. Dixon SL, Merz KM Jr, (1996) J. Chem. Phys. 104: 6643

5. Boys SF, (1960) Rev. Mod. Phys. 32: 296

6. Edmiston C, Ruedenberg K, (1965) J. Chem. Phys. 43: S97

7. Pipek J, Mezey PG, (1989) J. Chem. Phys. 90: 4916

8. Naray G - Szabo, (2000) Comput. Chem. 24: 287

9. Angyan JG, Naray G - Szabo, (1991) in Theoretical Models of Chemical Bonding, Part 4, Edited by Z.B.Maksic, Springer-Verlag, pag 1

10. Smits GF, Altona C, (1985) 67: 461

11. McWeeny R, (1992) Methods of molecular quantum mechanics, Academic Press

12. Stoll H, Wagenblast G, Preuss H, (1980) Theoret. Chim. Acta 57: 169

13. Sironi M, Famulari A, (1999) Theor. Chem. Acc. 103: 417

14. Fornili A, Sironi M, Raimondi M, (in press) Theochem

15. Szekeres Z, Surjan PR, (2003) Chem. Phys. Letts 369: 125

16. Couty M, Bayse CA, Hall MB, (1997) Theor. Chem. Acc. 97: 96

17. Gianinetti E, Raimondi M, Tornagli E, (1996) Int. J. Quantum Chem. 60: 157

18. Burresi E, Sironi M (submitted)

19. Sironi M, Famulari A, Raimondi M, Chiesa S, (2000) J. Mol. Struct. (Theochem) 47: 529

20. Assfeld X, Rivail JL, (1996) Chem. Phys. Lett. 263: 100

21. Ferré N, Assfeld X, Rivail JL, (2002) J. Comput. Chem. 23: 610

22. Pulay P, (1983) Chem. Phys. Letts 100: 151

23. Diner S, Malrieu JP, Claverie (1969) Theor. Chim. Acta 13: 1

24. Daudey JP, Diner S (1972) Int. J. Quant. Chem. 6: 575

25. Bonaccorsi R, Petrongolo C, Scrocco E, Tomasi J (1969) Theor. Chim. Acta 15: 332

26. Löwdin PO, (1955) Phys. Rev. 97: 1474

27. McWeeny R, Ohno KA, (1960) Proc. Roy. Soc. A255: 367 
28. Granovsky Alex A, http://classic.chem.msu.su/gran/gamess/index.html

29. Schmidt MW, Baldridge KK, Boatz JA, Elbert ST, Gordon MS, Jensen JJ, Koseki S, Matsunaga N, Nguyen KA, Su S, Windus TL, Dupuis M, Montgomery JA, (1993) J. Comput. Chem. 14: 1347

30. A general purpose Valence Bond program, written by M. Raimondi Universita' di Milano 
Figure captions

Fig. 1 Definition of the molecular fragments for the butane molecule Fig. 2 Plots of two virtual ELMOs before (A) and after (B) the Gram-Schmidt orthogonalization described in the text

Fig. 3 Energy values of different ELMO-VB calculations with respect to the increasing number of single excitations . The empty (full) circle refers to the use of one (two) virtual orbitals.

Fig. 4 Percentage of the energy recovered from different ELMO-VB calculations with respect to the increasing number of single excitations. The $100 \%$ value refers to the inclusion of all the single excitations in the selected set of virtual orbitals. The empty (full) circle refers to the use of one (two) virtual orbitals. Fig. 5 Definition of the molecular fragments for the 3-pentanone molecule Fig. 6 The transferring of the ELMOs for the 3-pentanone molecule 
Table 1 - Energy values for the butane molecule ${ }^{\mathrm{a}}$

\begin{tabular}{lcc}
\hline Method & Energy (a.u.) & $\Delta \mathrm{E}(\mathrm{kcal} / \mathrm{mol})$ \\
\hline RHF & -157.23468353 & 0.00 \\
LMO & -157.10578937 & 80.88 \\
ELMO & -157.19015516 & 27.94 \\
ELMO-VB (1) & -157.20084621 & 21.23 \\
ELMO-VB (2) & -157.22766442 & 4.40 \\
ELMO-VB (Top_1) & -157.19903195 & 22.37 \\
ELMO-VB (Top_2) & -157.22499207 & 6.08 \\
\hline
\end{tabular}

${ }^{\mathrm{a}}$ The acronyms are described in the text 
Table 2 - Energy values for the 3-pentanone molecule ${ }^{\mathrm{a}}$

\begin{tabular}{lcc}
\hline Method & Energy (a.u.) & $\Delta \mathrm{E}(\mathrm{kcal} / \mathrm{mol})$ \\
\hline RHF & -269.91403081 & 0.00 \\
LMO & -269.71535498 & 124.67 \\
ELMO & -269.82754481 & 54.27 \\
ELMO-Transf & -269.82315703 & 57.02 \\
ELMO-VB (1) & -269.86551984 & 30.44 \\
ELMO-VB (2) & -269.89686563 & 10.77 \\
ELMO-VB (Top_1) & -269.85384479 & 37.77 \\
ELMO-VB (Top_2) & -269.89106528 & 14.41 \\
\hline
\end{tabular}

${ }^{a}$ The acronyms are described in the text 
Figure 1 -

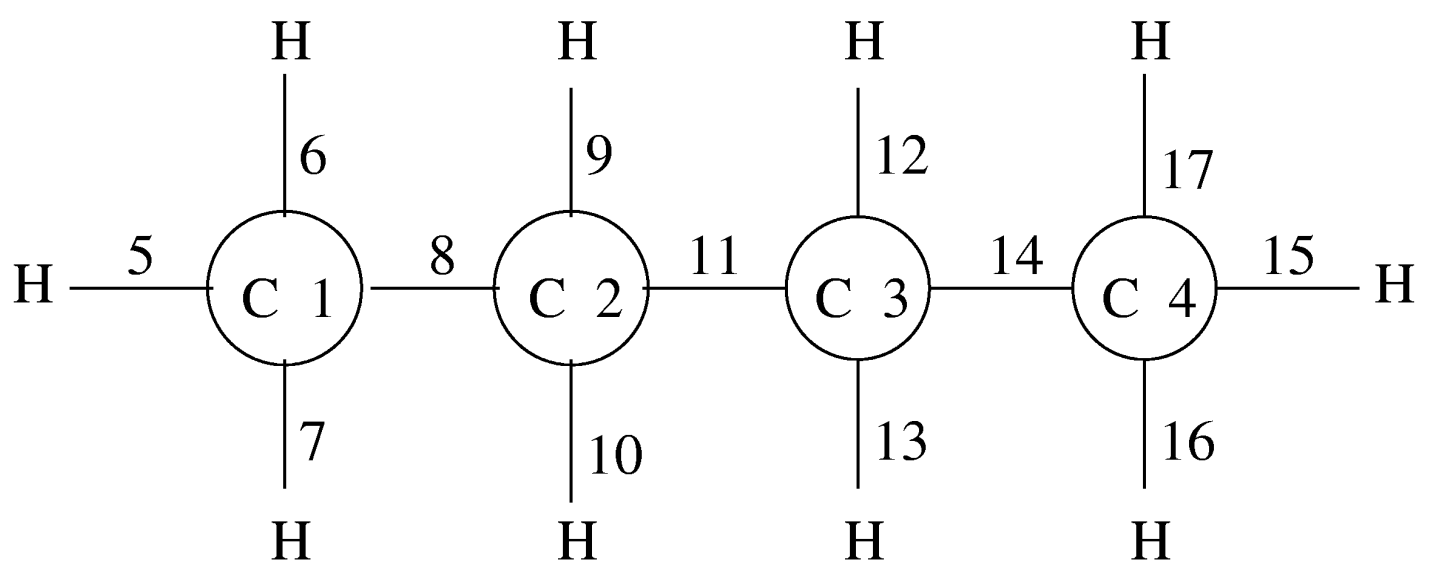


Fig. 2A
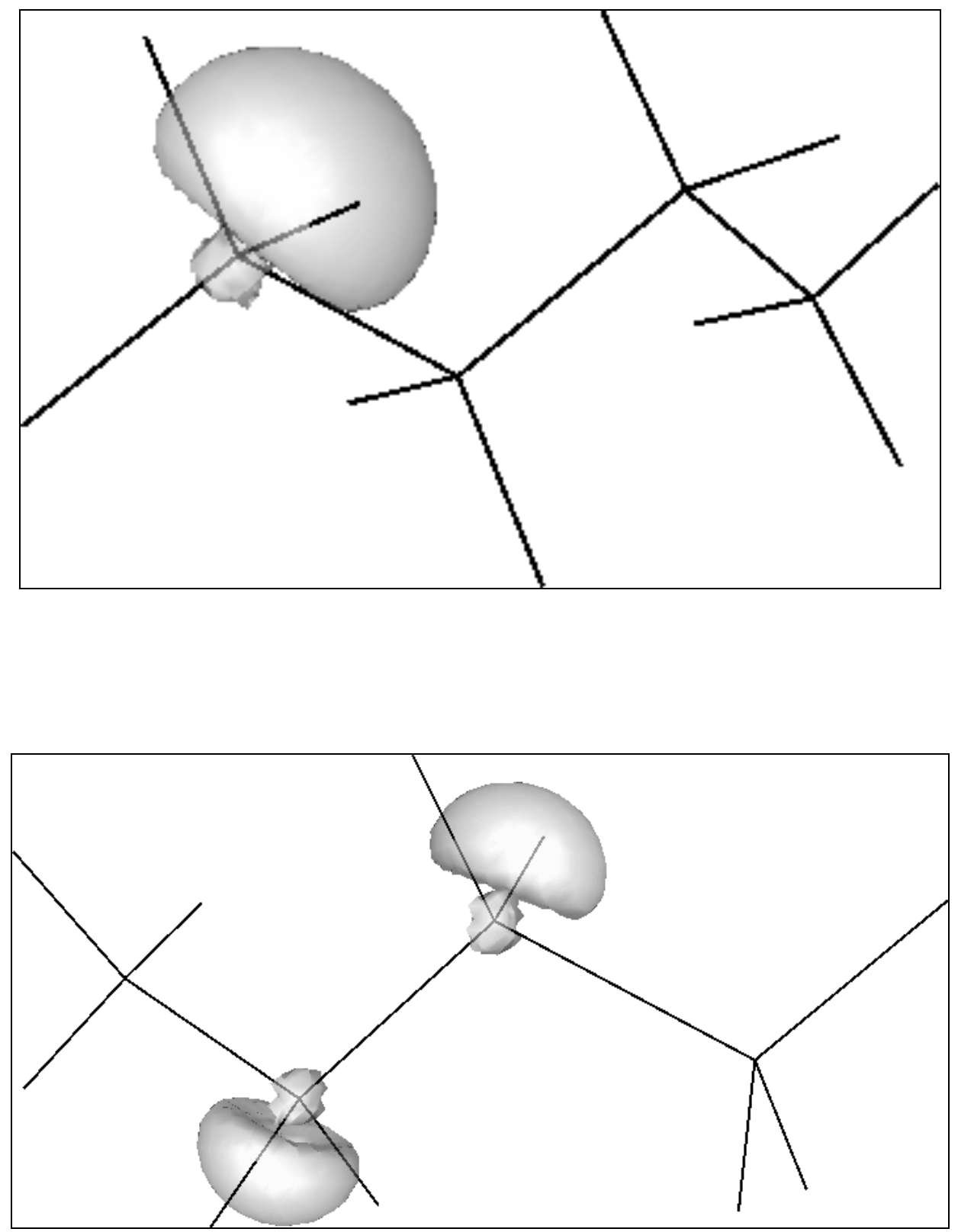
Fig. 2B
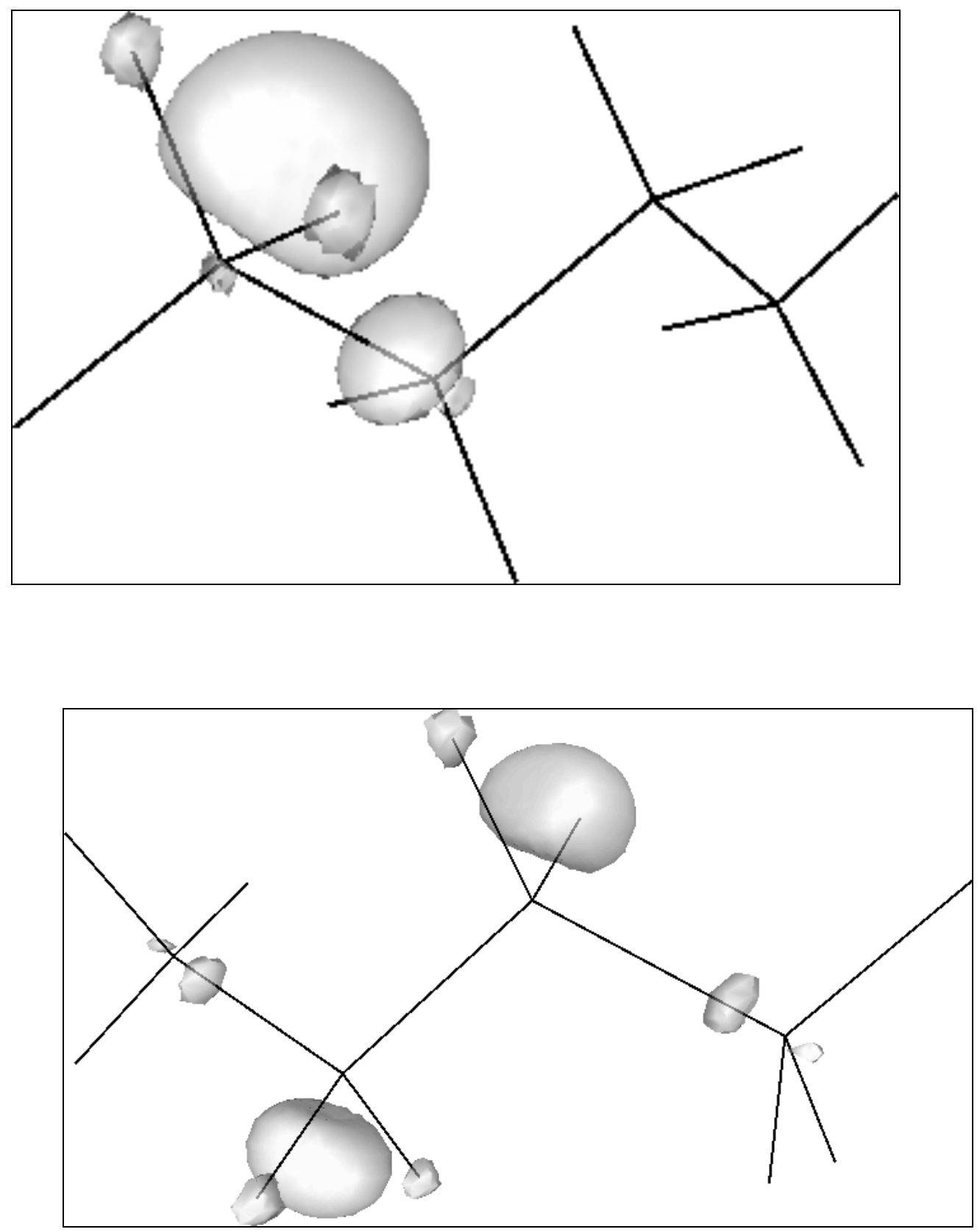
Fig. 3

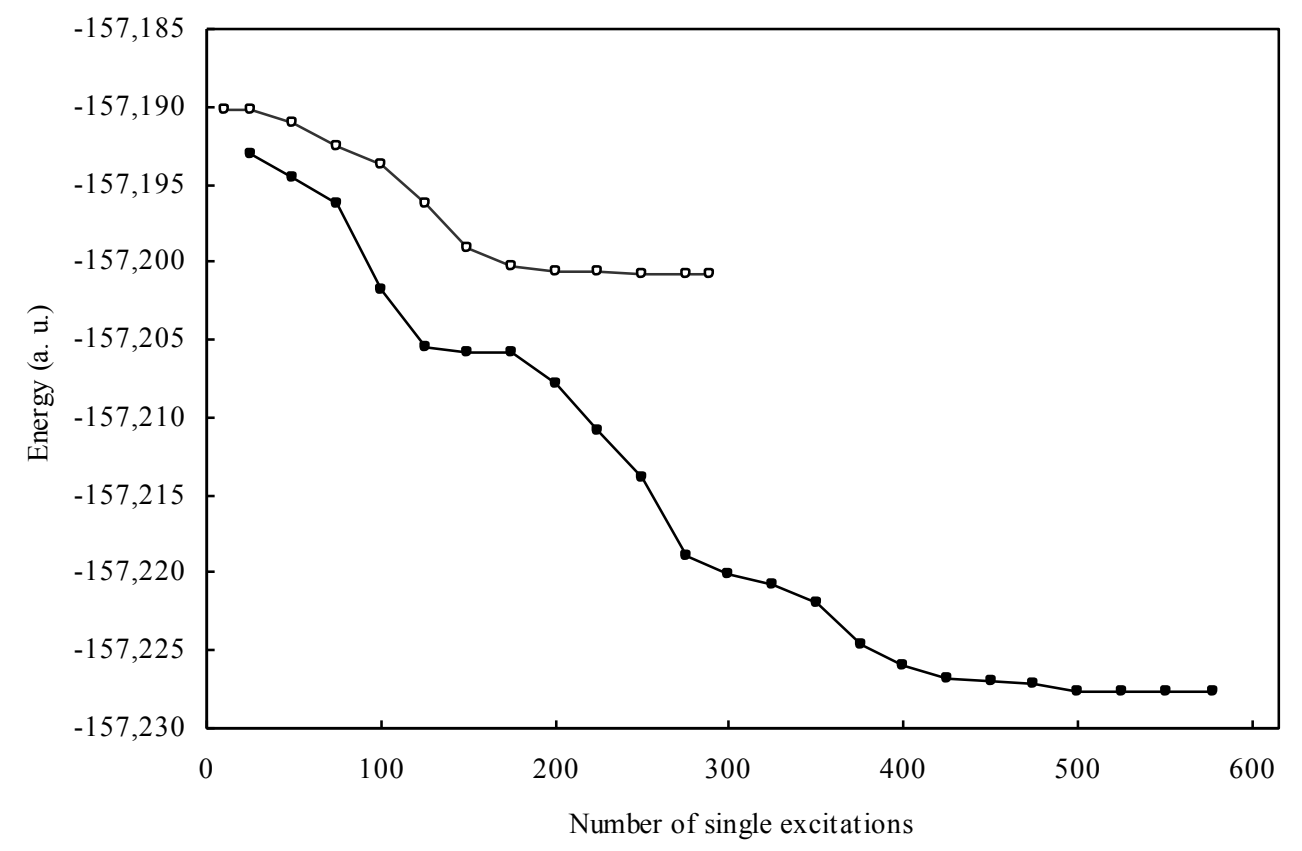


Fig. 4

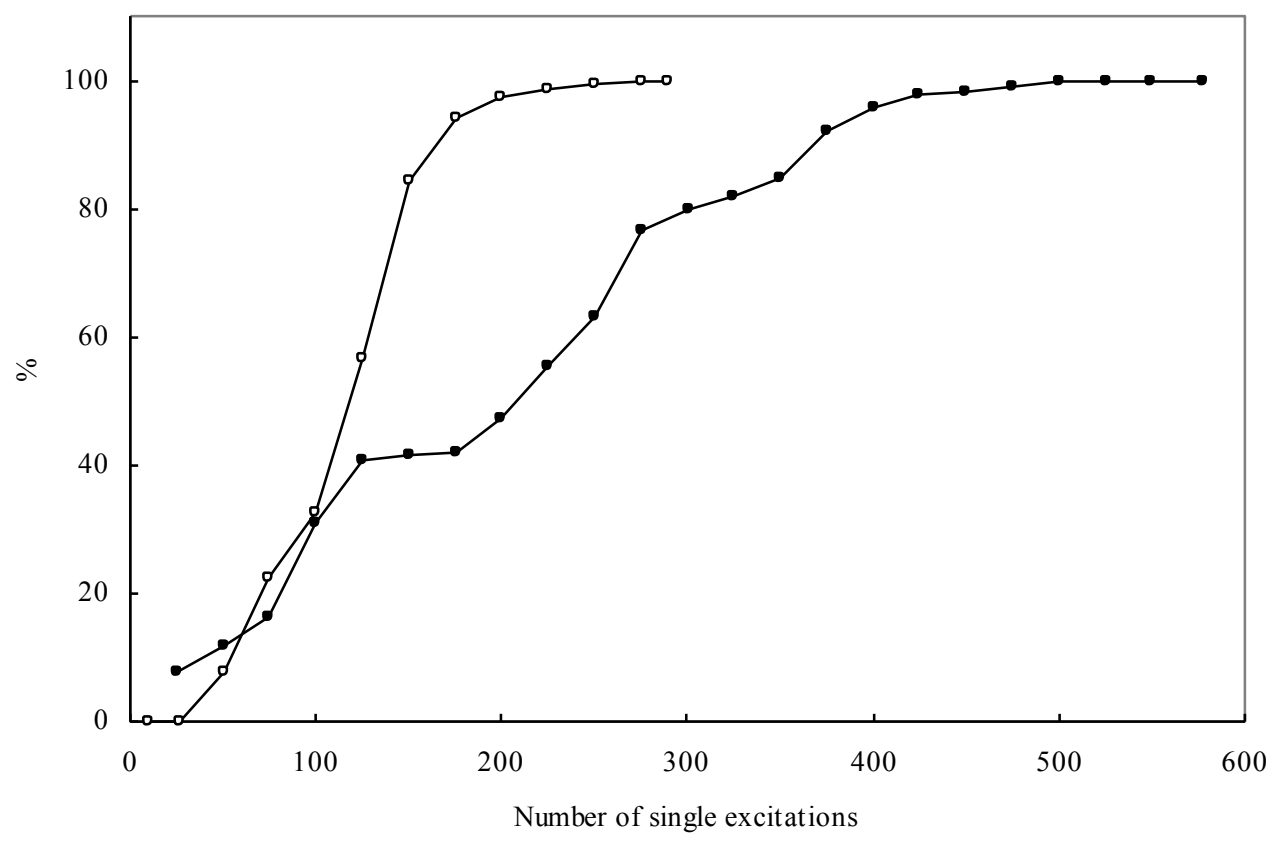


Fig. 5

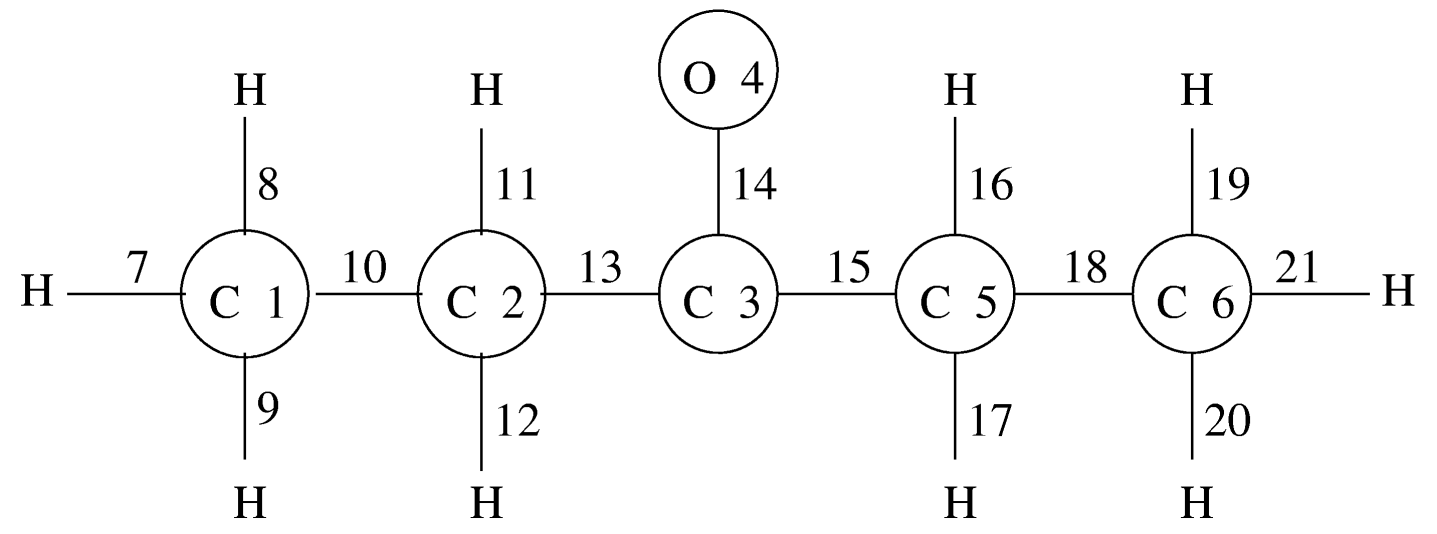


Fig. 6

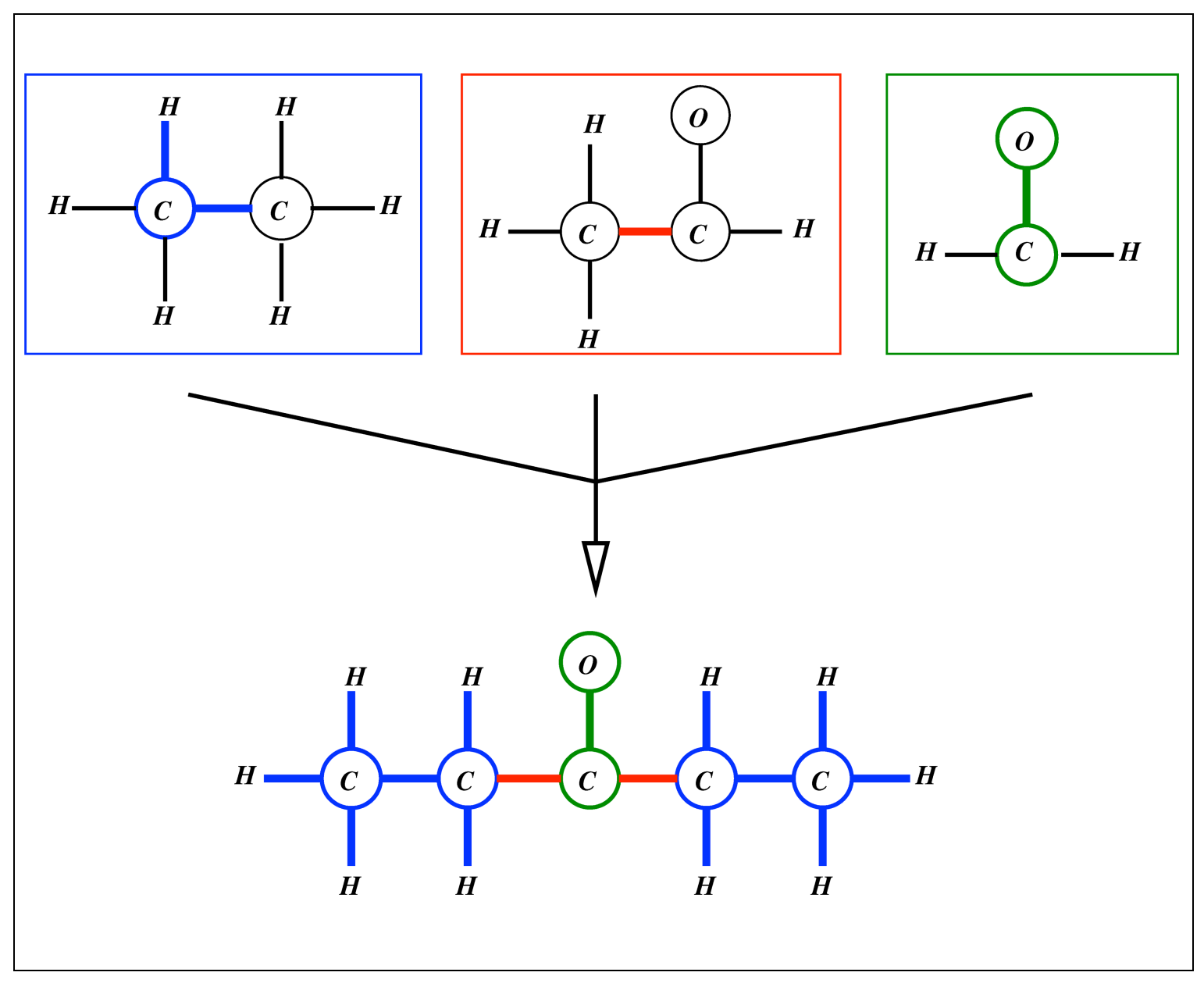

